\title{
Synaptonemal complex karyotype of zebrafish
}

\author{
BMN Wallace and $\mathrm{H}$ Wallace \\ School of Biosciences, University of Birmingham, Edgbaston, Birmingham B15 2TT, UK
}

\begin{abstract}
Meiotic cells of zebrafish have been prepared to show synaptonemal complexes (SCs) by light and electron microscopy. Completely paired SCs from both spermatocytes and oocytes were measured to produce an SC karyotype. The SC karyotype resembles the somatic karyotype of zebrafish and has no recognisable sex bivalent.
\end{abstract}

\begin{abstract}
Measurements of total SC length indicate that SCs grow longer and develop centromeres during pachytene. Oocytes consistently have longer SCs than spermatocytes, presumably correlated with the reported higher recombination frequency in females than in males.

Heredity (2003) 90, 136-140. doi:10.1038/sj.hdy.6800184
\end{abstract}

Keywords: synaptonemal complex; karyotype; sex chromosomes; zebrafish

\section{Introduction}

There are several descriptions of synaptonemal complex (SC) formation during meiotic prophase of teleost fishes, almost exclusively from spermatocytes. They include comparative studies of a few of the 20000 or so teleost species and more specific studies, such as investigations of the pairing behaviour of sex chromosomes (Yajuan and Qixing, 1991; Peterson et al, 1994; Oliveira et al, 1995; Toledo and Foresti, 2001; Campos-Ramos et al, 2001; Traut and Winking, 2001).

The somatic karyotype of zebrafish (Danio rerio) has been examined repeatedly as a counterpart to the extensive genetic analysis of this species, reviewed by Sola and Gornung (2001). The diploid number of 50 chromosomes, which can be distinguished by replication banding (Amores and Postlethwaite, 1999), does not include any recognisable sex chromosomes. Traut and Winking (2001) have provided the only information on meiosis in zebrafish. They measured SCs from spermatocytes and found no indication of a heteromorphic sex chromosome pair.

We have obtained SC preparations from both sexes of zebrafish, which seems necessary in any search for sex chromosomes as a heteromorphic pair might occur in either sex - both $\mathrm{XX} / \mathrm{XY}$ and $\mathrm{ZZ} / \mathrm{ZW}$ mechanisms of sex determination exist among teleosts in addition to various environmental sex determination mechanisms (Baroiller et al, 1999). We find that the SC karyotypes of both sexes of zebrafish conform to the somatic karyotype, but the total SC length in oocytes is considerably greater than in spermatocytes. We argue that zebrafish do not have a genetic means of sex determination and thus cannot possess sex chromosomes, and that the difference in SC length at pachytene of meiosis reflects the different recombination frequencies of male and female zebrafish.

Correspondence: H Wallace, School of Biosciences, University of Birmingham, Edgbaston, Birmingham B15 2TT, UK.

E-mail: H.Wallace@bham.ac.uk

Received 11 February 2002; accepted 3 August 2002

\section{Materials and methods}

Zebrafish were purchased locally and kept under standard conditions (Westerfield, 1995). Ovaries were dissected from young females, $25-30 \mathrm{~mm}$ long, before most oocytes had entered the growth phase. Testes were obtained from young adult males with relatively few mature sperm. Ovaries collected from about 10 specimens or a pair of testes were treated in exactly the same way, by maceration with a plastic pestle in a microfuge tube with enough cold half-strength Ham's F10 tissue culture medium to make a thick suspension. After allowing large aggregates to settle for $6 \mathrm{~min}$, one drop of the supernatant was added to two drops of detergent $0.03 \%$ Joy (Proctor and Gamble) pH 8.4 on a microscope slide and gently mixed. After allowing nuclei to settle for $6 \mathrm{~min}$, five drops of $4 \%$ paraformaldehyde were added and the mixture was dried down at $30^{\circ} \mathrm{C}$ overnight. Further processing and staining with silver nitrate have been described previously (Wallace and Hultén 1985; Wallace et al, 1992). Some preparations were made for light microscopy, while others used slides coated with a plastic film for electron microscopy. The film was produced by dipping a slide in 1\% Optilux (Falcon Petri dish fragments) in chloroform and then allowing it to dry. The film was later floated off and H6 Maxtaform copper grids were placed over identified stained cells.

Synaptonemal complexes (SCs) in 17 spermatocytes and 11 oocytes were measured from tracings of photographs (magnified about $\times 4000$ for light or $\times 5-6000$ for electron microscopy), using a video camera and Digihurst Image Analysis computer program. The karyotype was constructed by standard procedures which have been explained in more detail and justified elsewhere (Denver, 1960; Levan et al, 1964; Croft and Jones, 1986; Wallace, 1996). Each measurement was converted to a relative value (a percentage of the total SC length of that nucleus). The relative lengths of SCs in each nucleus were assigned a rank order, from longest to shortest, and the lengths of SCs occupying each rank position were averaged across nuclei. Centromere indices (short arm as 
fraction of the whole SC length) were assigned by their modal positions in the data set.

\section{Results}

Silver-stained spread preparations mainly reveal axial cores and lateral elements of the SC, and incidental features such as nucleoli and sperm tails. Our observations correspond to the sequence described in trout (Oliveira et al, 1995). Zygotene is recognised by about 50 incompletely paired stretches of lateral elements which usually carried an attachment plaque at one end (Figure 1a). Pairing apparently begins exclusively at the ends of each bivalent, before each axial core is complete, and extends towards the middle. No centromeres are evident at this stage. Pachytene is defined by the complete pairing of all bivalents, so that 25 SCs are present in each spread preparation (Figure 1b, c). We have found only one example of a late pairing segment in an oocyte that otherwise had completely formed SCs, some showing centromeres (Figure 1b). The unpaired axial cores represent most of the long arm of the fourth largest bivalent. We have also found several instances of the anomalous SC described by Traut and Winking (2001) as being fully paired and symmetrical but decorated with silver-stained excrescences. The clearest example in our material showed these decorations on the long arm of the sixth largest SC (Figure 1c).

Centromeres were only present on some definitive pachytene spreads with completely paired SCs, so we presume they develop in mid- or late-pachytene. Diplotene was rarely encountered in our preparations. The SC disintegrates and the lateral elements disappear, eventually resulting in unusual spermatocyte nuclei (previously noticed in grasshopper spermatocytes by Dr Juan Santos, personal communication) which might

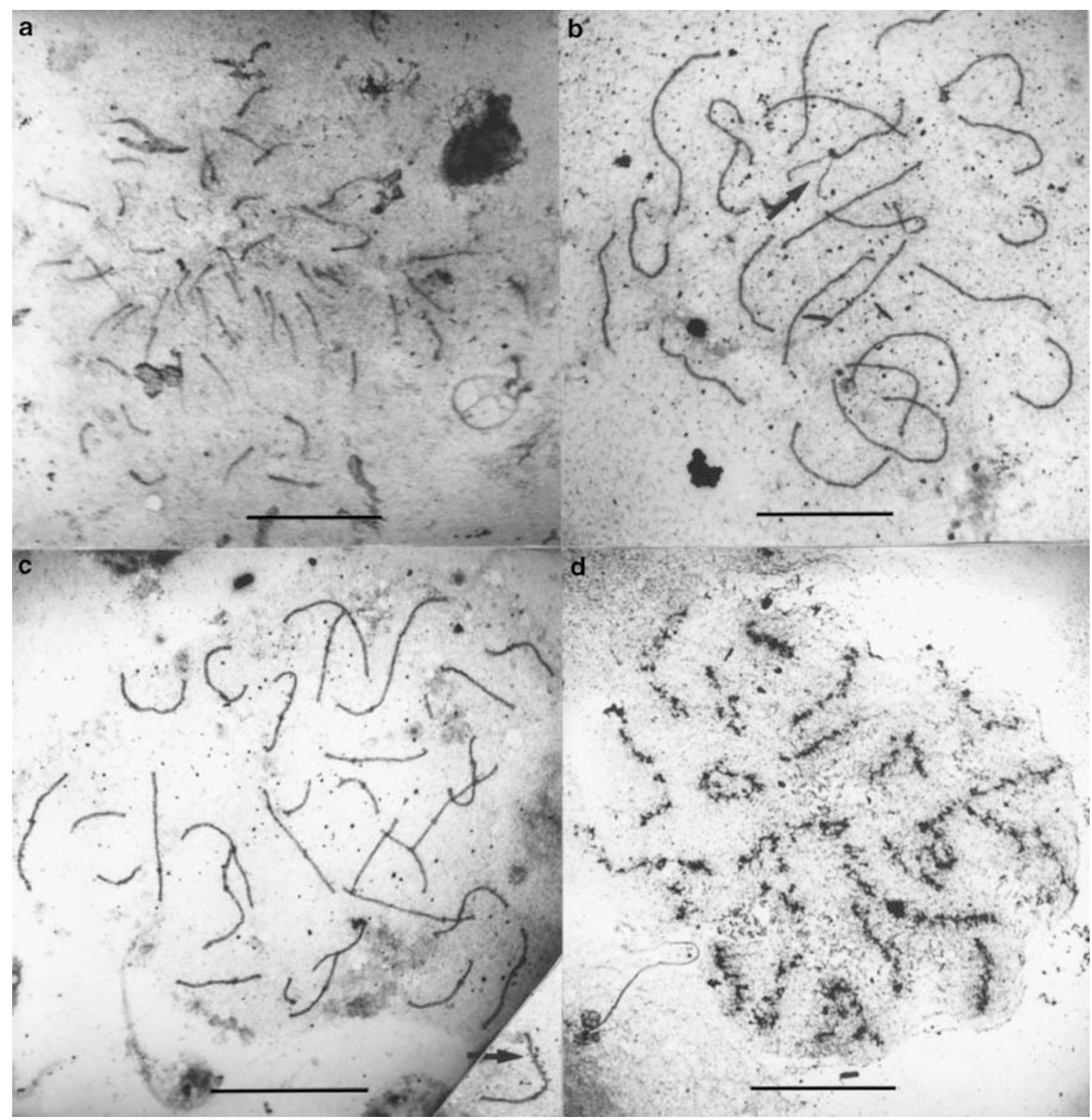

Figure 1 Electron micrographs of zebrafish meiosis. (a) Zygotene oocyte, showing 50 stretches of SC with an attachment plaque at one of each. (b) Pachytene oocyte with 24 completely paired bivalents and one bivalent unpaired for the distal half of the long arm (arrowed). (c) Pachytene spermatocyte with 25 completely paired bivalents. The prominent centromeres allowed easy reconstruction of the six breakpoints. Inset, an SC with excrescences on the long arm (arrowed). (d) Diplotene spermatocyte with 25 bivalents but little residual SC. Scale $\mathrm{bar}=10 \mu \mathrm{m}$. 
be diplotene or diakinesis (Figure 1d). This nucleus contained approximately 25 contracted bivalents, some in a cross or ring configuration suggesting one or two chiasmata. The bivalents lacked an axial support or chromatid core, and thus resembled those described by Rufas et al (1992) at diakinesis in Chorthippus. Oocyte nuclei did not reach this stage in our immature fish, but grew considerably with a typical diplotene appearance of diffuse chromatin and multiple nucleoli.

\section{Karyotype}

Measurements of complete sets of pachytene SCs show 25 bivalents that can be arranged by size, conforming closely to the measurements previously obtained from male zebrafish (Traut and Winking, 2001). Measured sets of SCs with visible centromeres allowed us to construct an SC karyotype (Figure 2) and thus compare our results to the published somatic karyotypes. The relative lengths for spermatocyte and oocyte SCs correspond well with each other and reasonably well with somatic chromosome lengths, but the absolute lengths differ. Spermatocyte SCs (Figure 2) are about 4 times as long as somatic metaphase chromosomes and oocyte SCs are usually even longer. Although greater length should provide greater accuracy of measurement, the centromere positions suggest the SC karyotype does not allow identification of most individual bivalents. For instance, the longest metacentric bivalent, No. 5, 6 or 7 of the somatic karyotype (Pijnacker and Ferwerda, 1995; Daga et al, 1996; Amores and Postlethwaite, 1999; Sola and Gornung, 2001), is No. 7 of our SC karyotype but sometimes occurs as 5-9 in order of size. Ranking the intermediatesized SCs probably imposes a spurious consistency, considering that the somatic karyotypes show groups of 2-7 chromosomes as being of apparently identical length. The only other metacentric, No. 11 of the SC karyotype (Figure 2) and of the somatic karyotype reported by Gornung et al (1997), is likely to be identical to chromosome 15 of the replication-banded somatic karyotype (Amores and Postlethwaite, 1999). Remnants of nucleoli have been found in several preparations attached to the end of the long arms of bivalent 1,2 or 3, confirming results from somatic karyotyping (Daga et al, 1996; Gornung et al, 1997).

The SC karyotype appears to be identical in both sexes but there is considerable variation in the total length of the SC complement. The SCs of oocytes were consistently longer than those of spermatocytes (Table 1) and, within each sex, pachytene spreads showing centromeres were consistently longer than spreads which lacked centromeres. The differences are small but significant for spermatocytes, while the massive extension and variability of oocyte SCs probably indicate that the oocyte growth phase has begun during pachytene.

\section{Discussion}

According to our limited observations on the development and later destruction of the synaptonemal complex, meiosis in zebrafish conforms to the general sequence of events described in other vertebrates. Several features of the silver-stained preparations recorded in the results occurred sporadically in some nuclei with clear SCs but

Table 1 Total SC lengths of zebrafish pachytene cells in $\mu \mathrm{m}$, mean \pm SD (no. of cells)

\begin{tabular}{lcc}
\hline & Spermatocytes & Oocytes \\
\hline SCs with centromeres & $197.5 \pm 28.0(9)$ & $324.3 \pm 63.6(6)$ \\
SCs without centromeres & $173.9 \pm 11.6(8)$ & $198.7 \pm 18.1(5)$ \\
\hline
\end{tabular}

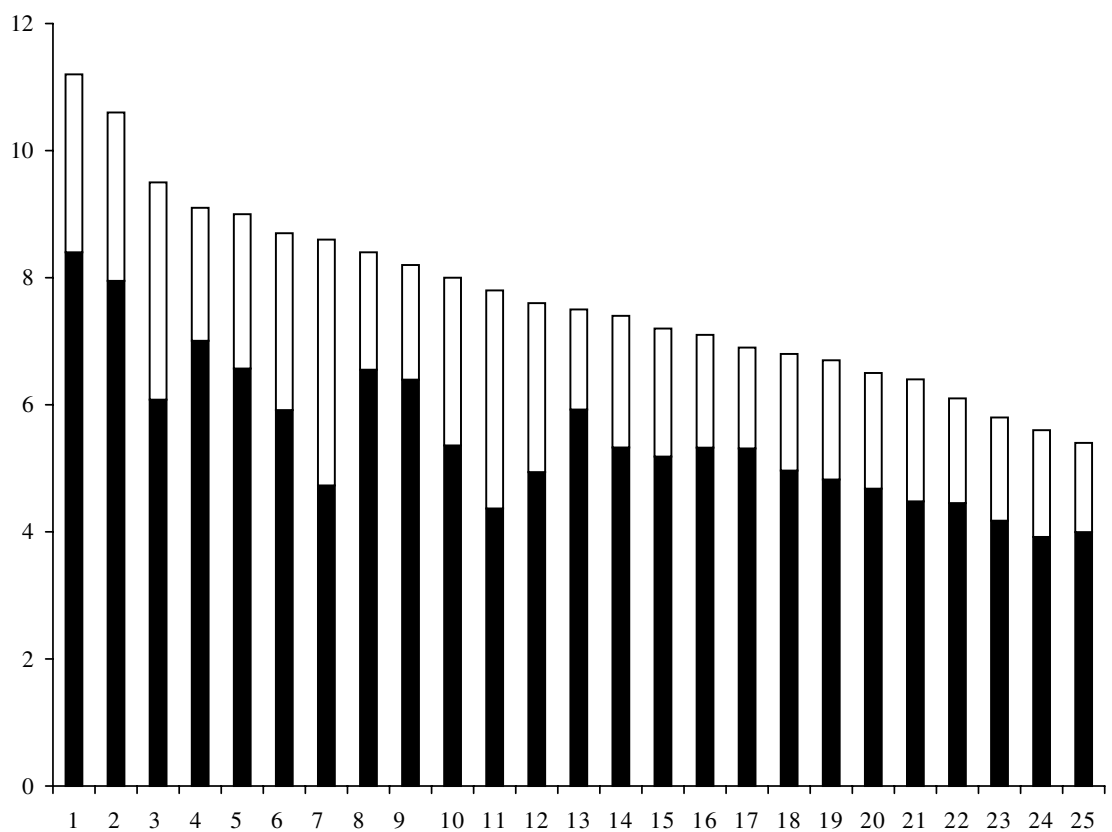

Figure 2 Idiogram of the SC karyotype of zebrafish. Ordinate, mean length in $\mu \mathrm{m}$ of each SC size class from spermatocytes showing centromeres. Abscissa, SCs arranged in order of decreasing size. Centromeres are shown in the modal position for each size class, long arms as solid columns and short arms as open columns. 
not in others. These include the centromeres, nucleoli and the 'excrescences' shown in Figure 1c.

Centromeres were detected as a thickening of the SC which probably represents staining of attached protein. They were usually present on all 25 SCs of a nucleus, or none, and nuclei showing centromeres had a greater total SC length than those without centromeres. We interpret these observations as a developmental sequence in both spermatocytes and oocytes. Early pachytene stages show complete SCs but no centromeres, which only develop at mid or late pachytene as the bivalents become less condensed and longer. This is a prelude to the typical nuclear growth and chromatin decondensation at diplotene, occurring to a limited extent in spermatocytes but persisting longer and to a massive degree in the oocytes of all oviparous vertebrates.

Nucleoli were usually dispersed by the spreading procedure in zebrafish pachytene nuclei, so only occasional nuclei retained a mass of nucleolar material attached to an SC. The attachment sites recorded in the nuclei used for karyotyping were always at the end of the long arm of bivalent 1, 2 or 3 . These sites had previously been identified as nucleolar organisers in the somatic karyotype by silver staining (Daga et al, 1996) or by in situ hybridisation (Gornung et al, 1997). The multiple nucleoli noticed in diplotene oocytes are presumably not attached to any chromosomal site.

The excrescences occasionally present on the long arm of one SC have been recorded as a regular feature of zebrafish spermatocytes by Traut and Winking (2001), who found them on bivalent 3,4 or 5 and suggested that the decorated region corresponded to the heterochromatic long arm of chromosome 3 in the somatic karyotype (Pijnacker and Ferwerda, 1995). Similar excrescences have been recorded on unpaired regions of the XY bivalent in spermatocytes of several mammals, for instance by Solari (1994). They are displayed by shrew but not by mouse spermatocytes (Wallace and Searle, 1990; Wallace et al, 1992) and might well be composed of unused SC precursors in the sex vesicle. On the other hand, the zebrafish excrescences occur on a completely paired bivalent and Traut and Winking (2001) also reported a decorated autosomal SC in guppy spermatocytes. We do not attach any significance to our failure to find a decorated SC in oocytes, as there were only two or three clear examples in the zebrafish spermatocytes.

The 25 completely paired SCs correspond to the 25 bivalents expected from the somatic karyotype. Neither sex shows consistently unpaired or late pairing segments of SC, which have been recognised in other vertebrates and reasonably interpreted as indications of sex chromosomes. Our failure to identify a sex bivalent in zebrafish does not demonstrate its absence, of course, but was the expected result in view of the evidence that zebrafish probably do not employ a genetic means of sex determination. No sex linkage has been reported for several thousand mapped loci. Both androgenetic and gynogenetic clones produce offspring of both sexes (Streisinger et al, 1981; Corley-Smith et al, 1996) which eliminate $\mathrm{XX} / \mathrm{XY}$ and $\mathrm{ZZ} / \mathrm{ZW}$ mechanisms of sex determination (Wallace et al, 1999). The variable sex ratios obtained from these clones and normal matings have led to the suggestion that zebrafish reared in poor conditions become male (Pelegri and Schulte-Merker,
1999) or that some unknown environmental condition acts as a sex determinant. We have reared zebrafish at various temperatures from 20 to $32^{\circ} \mathrm{C}$ and found no evidence for temperature-dependent sex determination. We are intrigued by the possibility that the last round of genomic doubling in zebrafish evolution, as documented by Postlethwaite et al (1999), might have disrupted an ancestral genetic sex determination mechanism leaving relics of sex chromosomes. The features of chromosome 3 mentioned above would make it a candidate relict sex chromosome, like chromosome 1 of crested newts (Wallace et al, 1997).

The total length of SC at pachytene given in Table 1 is within the range of values obtained for other teleosts (Peterson et al, 1994) and that for spermatocytes is virtually identical to the results of Traut and Winking (2001). Assuming a haploid DNA content of 1.8 pg (Sola and Gornung, 2001), the packing ratio of zebrafish SCs of $5-10 \mathrm{pg} \mathrm{DNA} / \mathrm{mm}$ is also very similar to that calculated for trout (Oliveira et al, 1995). The zebrafish provides an example of higher recombination frequency in females than in males, estimated at 2900 and $1700 \mathrm{cM}$, respectively (Postlethwaite et al, 1999). A single chiasma on each bivalent would give a theoretical total frequency of $1250 \mathrm{cM}$, so spermatocyte bivalents are expected to carry one or two chiasmata and oocyte bivalents are likely to carry two or three. Traut and Winking (2001) have confirmed this expectation for spermatocytes but the frequency of oocyte chiasmata is still unknown.

The greater lengths of SC or lower DNA packing ratios of zebrafish oocytes relative to spermatocytes thus correlate with their higher recombination frequency. Peterson et al (1994) obtained comparative data to support their suggestion that this correlation might apply to all veretebrates. We also believe it is generally true, although Triturus cristatus provides an exception to it (Wallace and Wallace, 1995). Our observation here that fully paired SCs of early pachytene are still capable of appreciable further growth, especially in oocytes, means that SC measurements and SC packing ratios are subject to even greater errors than we had previously appreciated.

\section{Acknowledgements}

We thank Drs Walther Traut and Heinz Winking for their advice and a proof copy of their article, and Stuart Grice for some of the measurements in Table 1.

\section{References}

Amores A, Postlethwaite JH (1999). Banded chromosomes and the zebrafish karyotype. Methods Cell Biol 60: 323-338.

Baroiller J-F, Guiguen Y, Fostier A (1999). Endocrine and environmental aspects of sex differentiation in fish. Cell Mol Life Sci 55: 910-931.

Campos-Ramos R, Harvey SC, Masambanda JS, Carrasco LAP, Griffin DK, Mcandrew BJ et al (2001). Identification of putative sex chromosomes in the blue tilapia, Oreochromis aureus, through synaptonemal complex and FISH analysis. Genetica 111: 143-153.

Corley-Smith GE, Lim CTJ, Brandhorst BP (1996). Production of androgenetic zebrafish (Danio rerio). Genetics 142: 1265-1276.

Croft JA, Jones GH (1986). Surface spreading of synaptonemal complexes in locusts. I. Pachytene observations. Chromosoma 93: 483-488. 
Daga RR, Thode G, Amores A (1996). Chromosome complement C-banding, Ag-NOR and replication banding in the zebrafish Danio rerio. Chromosome Res 4: 29-32.

Denver Study Group (1960). A proposed standard system of nomenclature of human mitotic chromosomes. Acta Genet: 10: 322-328.

Gornung E, Gabrielli I, Cataudella S, Sola L (1997). CMA3banding pattern and fluorescence in situ hybridization with 18s rRNA genes in zebrafish chromosomes. Chromosome Res 5: 40-46.

Levan A, Fredga K, Sandberg AA (1964). Nomenclature for centromeric position on chromosomes. Hereditas 52: 201-220.

Oliveira C, Foresti F, Rigolono MG, Tabata YA (1995) Synaptonemal complex analysis in spermatocytes and oocytes of the rainbow trout, Oncorhynchus mykiss (Pisces, Salmonidae) - the process of autosome and sex-chromosome synapsis. Chromosome Res 3: 182-190.

Pelegri F, Schulte-Merker S (1999). A gynogenesis based screen for maternal effect genes in the zebrafish, Danio rerio. Methods Cell Biol 60: 1-20.

Peterson DG, Stack SM, Healy JL, Donohoe BS, Anderson LK (1994). The relationship between synaptonemal complex length and genome size in four vertebrate classes (Osteichthyes, Reptilia, Aves, Mammalia). Chromosome Res 2: 153-162.

Pijnacker L, Ferwerda M (1995). Zebrafish chromosome banding. Genome 38: 1052-1055.

Postlethwaite J, Amores A, Force A, Yan Y-L (1999). The zebrafish genome. Methods Cell Biol 60: 149-163.

Rufas JS, Santos JL, Diez M, Suja JA (1992). Meiotic chromosome structure: relationship between the synaptonemal complex and the chromatid cores. Genome 35: 1054-1061.

Sola L, Gornung E (2001). Classical and molecular cytogenetics of the zebrafish, Danio rerio (Cyprinidae, Cypriniformes): an overview. Genetica 111: 397-412.

Solari AJ (1994). Sex Chromosomes and Sex Determination in Vertebrates. CRC Press: Boca Raton, FL. 308 pp.
Streisinger G, Walker C, Dower N, Knauber D, Singer F (1981). Production of clones of homozygous diploid zebra fish (Brachydaniorerio). Nature 291: 293-296.

Toledo LFD, Foresti F (2001). Morphologically differentiated sex chromosomes in neotropical freshwater fish. Genetica 111: 91100.

Traut W, Winking H (2001). Meiotic chromosomes and stages of sex chromosome evolution in fish: zebrafish, platyfish and guppy. Chromosome Res 9: 659-672.

Wallace BMN (1996). A pachytene karyotype of the common shrew (Sorex araneus). Hereditas 125: 219-223.

Wallace BMN, Hultén MA (1985). Meiotic chromosome pairing in the normal human female. Ann Hum Genet 49: 215-226.

Wallace BMN, Searle JB (1990). Synaptonemal complex studies of the common shrew (Sorex araneus). Comparison of Robertsonian heterozygotes and homozygotes by light microscopy. Heredity 65: 359-367.

Wallace BMN, Searle JB, Everett CA (1992). Male meiosis and gametogenesis in wild house mice Mus musculus domesticus from a chromosomal hybrid zone; a comparison between 'simple' Robertsonian heterozygotes and homozygotes. Cytogenet. Cell Genet 61: 211-220.

Wallace H, Wallace BMN (1995). Complete meiotic pairing of crested newt chromosomes. Genome 38: 1105-1111.

Wallace H, Badawy GMI, Wallace BMN (1999). Amphibian sex determination and sex reversal. Cell Mol Life Sci 55: 901-909.

Wallace H, Wallace BMN, Badawy GMI (1997). Lampbrush chromosomes and chiasmata of sex-reversed crested newts. Chromosoma 106: 526-533.

Westerfield M (1995). The Zebrafish Book, 3rd edn. University of Oregon Press: Oregon.

Yajuan L, Qixing Y (1991). Electron microscopic observation of synaptonemal complexes in six species of fishes. Chinese J Genet 18: 273-282. 